\title{
Early-life determinants of hypoxia-inducible factor 3A gene (HIF3A) methylation: a birth cohort study
}

\author{
Toby Mansell ${ }^{1,2}$ (D), Anne-Louise Ponsonby ${ }^{1,2,3}$, Vania Januar ${ }^{1}$, Boris Novakovic ${ }^{1,2}$, Fiona Collier ${ }^{1,4,5}$, David Burgner ${ }^{1,2,6}$,
} Peter Vuillermin ${ }^{1,4,5}$, Joanne Ryan ${ }^{1,7}$, Richard Saffery ${ }^{1,2^{*}}$ and Barwon Infant Study Investigator Team

\begin{abstract}
Background: Methylation of the hypoxia-inducible factor $3 a$ gene (HIF3A) has been linked to pregnancy exposures, infant adiposity and later BMI. Genetic variation influences HIF3A methylation levels and may modify these relationships. However, data in very early life are limited, particularly in association with adverse pregnancy outcomes. We investigated the relationship between maternal and gestational factors, infant anthropometry, genetic variation and HIF3A DNA methylation in the Barwon Infant Study, a population-based birth cohort. Methylation of two previously studied regions of HIF3A were tested in the cord blood mononuclear cells of 938 infants.

Results: No compelling evidence was found of an association between birth weight, adiposity or maternal gestational diabetes with methylation at the most widely studied HIF3A region. Male sex $(-4.3 \%, p<0.001)$ and pre-eclampsia $(-$ $5.4 \%, p=0.02$ ) negatively associated with methylation at a second region of HIF3A; while positive associations were identified for gestational diabetes $(4.8 \%, p=0.01)$ and gestational age ( $1.2 \%$ increase per week, $p<0.001)$. HIF3A genetic variation also associated strongly with methylation at this region $(p<0.001)$.

Conclusions: Pre- and perinatal factors impact HIF3A methylation, including pre-eclampsia. This provides evidence that specific pregnancy complications, previously linked to adverse outcomes for both mother and child, impact the infant epigenome in a molecular pathway critical to several vascular and metabolic conditions. Further work is required to understand the mechanisms and clinical relevance, particularly the differing effects of in utero exposure to gestational diabetes or pre-eclampsia.
\end{abstract}

Keywords: HIF3A, DNA methylation, SNPs, Pregnancy, Infant, Gestational diabetes, Pre-eclampsia

\section{Background}

Evidence suggests that risk factors for a range of metabolic and cardiovascular diseases begin very early in life, including in utero [1]. In turn, the in utero environment is sensitive to maternal environmental exposures [2], potentially mediating these effects. This is described by the Developmental Origins of Health and Disease $(\mathrm{DOHaD})$ concept, which postulates that the early-life environment is important in shaping later adult health and risk of disease [3].

\footnotetext{
* Correspondence: richard.saffery@mcri.edu.au

${ }^{1}$ Murdoch Children's Research Institute, Parkville, Australia

2Department of Paediatrics, University of Melbourne, Parkville, Australia

Full list of author information is available at the end of the article
}

The biological mechanism(s) underlying the influence of prenatal exposures on neonatal health and adult disease are poorly understood but are thought to be mediated, at least in part, by epigenetic processes, including DNA methylation [2]. In adults, both genome-wide and gene-specific methylation have been associated with adiposity-related measures, including body mass index (BMI), waist circumference and levels of inflammatory markers [4-11]. A cross-sectional epigenome-wide association study (EWAS) in adult blood identified DNA methylation of three CpG sites in the first intron of some transcript variants of the hypoxia-inducible factor $3 \alpha$ (HIF-3 $\alpha$ ) gene (HIF3A) in association with BMI [6], subsequently replicated by two independent cross-sectional studies using adult blood $[12,13]$. 
The family of hypoxia-inducible factors, including HIF$3 \alpha$, are believed to play key roles in angiogenesis, metabolism and obesity [14-16] and variation in DNA methylation of the associated $\mathrm{CpG}$ sites (identified in adipose tissue and whole blood) has been linked with altered gene expression [6] and adiposity measures in several subsequent studies $[4,7-11,17-21]$. The majority of previous findings are cross-sectional and have investigated methylation in blood, adipose tissue, or umbilical cord (Additional file 1). However, one longitudinal study in children found evidence for early BMI predicting later HIF3A methylation in blood [19]. Emerging evidence also suggests an influence of gestational diabetes [22] and maternal pre-pregnancy BMI on cord blood methylation at a second HIF3A promoter region [19]. Despite these findings, the tissue specificity and direction of causality at the two regions of HIF3A in newborns remain generally unclear.

Here, we aimed to investigate (1) the relationship between maternal factors in pregnancy and HIF3A methylation at two gene regions, (2) the relationship between infant anthropometry and HIF3A methylation, (3) the influence of $H I F 3 A$ genetic variation on methylation, and (4) the dependence of each of these influences on HIF3A methylation levels.

\section{Results}

\section{Cohort characteristics and methylation data}

The mean age of mothers in this study at conception was 31.4 years (standard deviation (SD) 4.7) and mean pre-pregnancy BMI 25.3 (SD 5.3). The incidence of gestational diabetes (GDM) and pre-eclampsia was 5.0\% (40/800) and 2.9\% (27/934), respectively. Mean infant gestational age was 39.5 weeks (SD 1.4), mean birth weight $3559.6 \mathrm{~g}$ (SD 496.3), and 51.4\% (482/938) of infants were male. Sample characteristics are shown in Table 1. The distribution of methylation for each $\mathrm{CpG}$ unit and the averages for the two HIF3A regions investigated in this study (herein referred to as HIF3A.1 and HIF3A.2) are shown in Fig. 1. The mean average methylation level across HIF3A.1 was $70.3 \%$ (SD 4.5), with mean methylation of individual CpG units ranging from 59.5 to $80.8 \%$. HIF3A.2 was generally less methylated, with a mean average methylation across HIF3A.2 of 38.5\% (SD 9.7) and mean methylation of individual CpG units between 20.2 and 66.4\%. Data were approximately normal in distribution and within each region was strongly correlated $(p<0.0001$ for all pairwise correlations, Additional file 2). As such, subsequent analyses focussed on the average methylation across each region.

\section{Pre-eclampsia and gestational diabetes associated with average HIF3A.2 methylation}

In univariate analysis investigating the relationship between maternal factors and cord blood methylation, preeclampsia was associated with lower average methylation across HIF3A.2 (- 6.38\% methylation, 95\% confidence interval $(\mathrm{CI})-10.94,-1.82, p=0.006)$, while GDM was associated with higher average HIF3A.2 methylation $(3.53 \%$ increased methylation, 95\% CI $-0.43,7.49, p=0.08$ ) (Table 2, Fig. 2a, b). No maternal measures were associated with average HIF3A.1 methylation.

Table 1 Cohort characteristics for the HIF3A.1 and HIF3A.2 samples

\begin{tabular}{lll}
\hline Characteristics & HIF3A.1 sample $n=490$ & HIF3A.2 sample $n=938$ \\
\hline Maternal & Mean (SD) & Mean (SD) \\
Age at conception (years) & $31.7(4.4)$ & $31.4(4.7)$ \\
Pre-pregnancy BMI (kg/m ${ }^{2}$ ) & $25 .(5.1)$ & $25.3(5.3)$ \\
& $n(\%)$ & $n(\%)$ \\
Smoked during pregnancy (any) & $59(12.0)$ & $146(15.6)$ \\
Gestational diabetes & $17(3.9)$ & $40(5.0)$ \\
Pre-eclampsia & $17(3.5)$ & $27(2.9)$ \\
Infant & & \\
& Mean (SD) & Mean (SD) \\
Gestational age (weeks) & $39.5(1.4)$ & $39.5(1.4)$ \\
Birth weight (g) & $3548.5(500.0)$ & $3559.6(496.3)$ \\
Birth weight z-score & $0.4(0.9)$ & $0.4(0.9)$ \\
Triceps+subscapular sum (mm) & $9.7(2.1)$ & $9.9(2.2)$ \\
Sex (male) & $n(\%)$ & $n(\%)$
\end{tabular}

SD standard deviation 


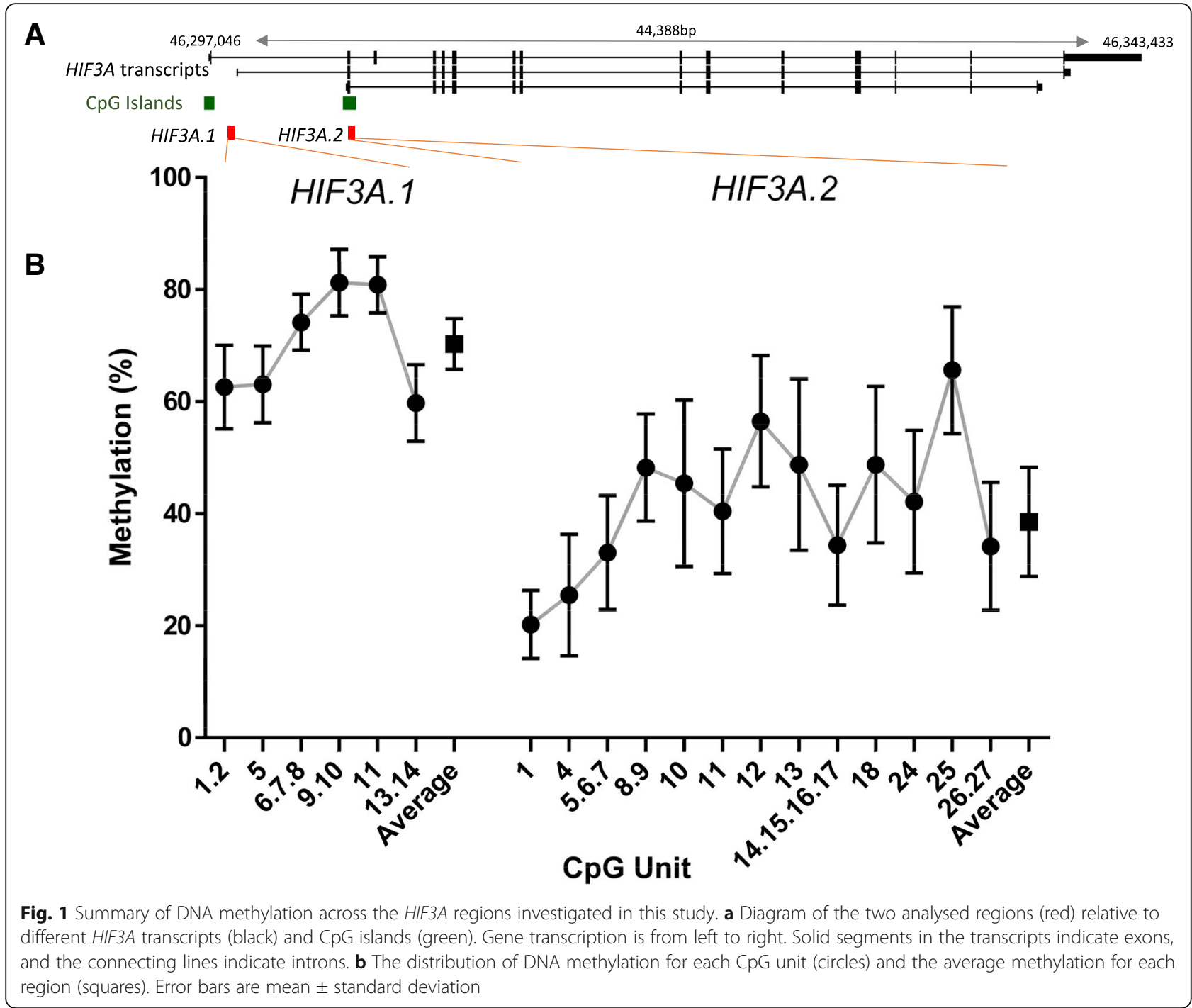

Infant sex and gestational age associate with average HIF3A.2 methylation; sex has weaker association with HIF3A.1 methylation

Male sex of the infant was negatively associated with average HIF3A.2 methylation (-3.87\% methylation compared to females, 95\% CI $-5.40,-2.34, p<0.001$ ) (Fig. 2c). Gestational age $(r=0.19, p<0.001)$ and absolute birth weight $(r=0.09, p=0.03)$ were positively associated with average HIF3A.2 methylation (Table 2). Infant sex had a weaker association with average methylation across HIF3A.1 (0.78\% increased methylation in male infants, $95 \% \mathrm{CI}-0.09,1.65, p=0.08)$.

\section{Genetic influences on HIF3A methylation stronger at HIF3A.2}

Of the 14 HIF3A tag SNPs considered in this analysis, rs3810298, rs112087991 and rs3826795 were strongly associated with average HIF3A.2 methylation, with genotype of rs3810298 showing the greatest effect $(\mathrm{C} / \mathrm{T}$ genotype $-8.48 \%$ compared to $\mathrm{C} / \mathrm{C}, 95 \% \mathrm{CI}-10.34$, 6.63, $p<0.001$; T/T genotype $-18.06 \%$ compared to $\mathrm{C} /$ C, 95\% CI $-23.65,-12.47, p<0.001$ ) (Table 3, Fig. 2d). All three of these SNPs were in strong linkage disequilibrium (Additional file 3). The genotype of rs8102595 was associated with average HIF3A.1 methylation (1.40\% increased methylation for heterozygote $\mathrm{A} / \mathrm{G}$ genotype compared to major allele homozygote A/A, 95\% CI 0.26, $2.54, p=0.02$ in linear regression model).

\section{Multivariable regression modelling for average HIF3A.2 methylation}

Given the multiple strong associations identified by univariate analyses, more detailed regression modelling was carried out for HIF3A.2. Key covariates of GDM, pre-eclampsia, gestational age, birth weight, infant sex and rs3810298 genotype all remained independently 
Table 2 Associations of cohort characteristics with average HIF3A.1 and HIF3A.2 methylation

\begin{tabular}{|c|c|c|c|c|}
\hline \multirow{2}{*}{$\frac{\text { Characteristics }}{\text { Maternal }}$} & \multicolumn{2}{|c|}{ Average HIF3A.1 methylation $n=423$} & \multicolumn{2}{|c|}{ Average HIF3A.2 methylation $n=609$} \\
\hline & & & & \\
\hline & $r$ & $p$ & $r$ & $p$ \\
\hline Age at conception (years) & 0.01 & 0.80 & -0.01 & 0.76 \\
\hline \multirow[t]{2}{*}{ Pre-pregnancy BMI (kg/m²) } & -0.01 & 0.81 & 0.04 & 0.40 \\
\hline & Effect (SE) & $p$ & Effect (SE) & $p$ \\
\hline Smoked during pregnancy (any) & $0.01(0.66)$ & 0.99 & $0.63(1.10)$ & 0.56 \\
\hline Gestational diabetes & $0.04(1.19)$ & 0.97 & $3.53(2.01)$ & 0.08 \\
\hline Pre-eclampsia & $0.09(1.24)$ & 0.94 & $-6.38(2.32)$ & 0.006 \\
\hline \multicolumn{5}{|l|}{ Infant } \\
\hline & $r$ & $p$ & $r$ & $p$ \\
\hline Gestational age (weeks) & -0.08 & 0.10 & 0.19 & $<0.001$ \\
\hline Birth weight (g) & -0.05 & 0.32 & 0.09 & 0.03 \\
\hline Birth weight z-score & -0.03 & 0.61 & 0.02 & 0.61 \\
\hline \multirow[t]{2}{*}{ Triceps+subscapular sum (mm) } & -0.01 & 0.89 & 0.03 & 0.54 \\
\hline & Effect (SE) & $p$ & Effect (SE) & $p$ \\
\hline Sex (male) & $0.78(0.44)$ & 0.08 & $-3.87(0.78)$ & $<0.001$ \\
\hline
\end{tabular}

Effect size given as difference in percentage methylation. $r$ correlation coefficient, $S E$ standard error. $p$ values for binary measures (smoking during pregnancy, gestational diabetes, pre-eclampsia, and male infant sex) are from unpaired two-tailed Student's $t$ tests comparing the exposed group to the unexposed group. $p$ values for continuous measures are from correlation coefficient tests

associated with average HIF3A.2 methylation (Table 4), with the exception of birth weight which attenuated after adjustment for gestational age and consequently was excluded from the final model. GDM $(4.62 \%$ increased methylation, 95\% CI 1.14, 8.09, $p=0.009$ ) and gestational age $(1.21 \%$ increased methylation per week, $95 \%$ CI $0.68,1.74, \mathrm{p}<0.001)$ were positively associated with methylation, whereas pre-eclampsia $(-5.20 \%, 95 \%$ CI $-9.51,-0.89, p=0.02)$ and male sex $(-3.77 \%$ compared to females, $95 \% \mathrm{CI}-5.26,-2.29, p<0.001)$ were negatively associated with methylation. A diagram of the associations is shown in Fig. 3. Genotype at rs3810298 was not associated with any of the maternal or infant measures included in the final HIF3A.2 linear regression model (Additional file 4).

\section{Additional sensitivity analyses}

Of the cellular proportions considered, granulocytes and, inversely, lymphocytes were associated with average HIF3A.2 methylation $(0.11 \%$ increased methylation
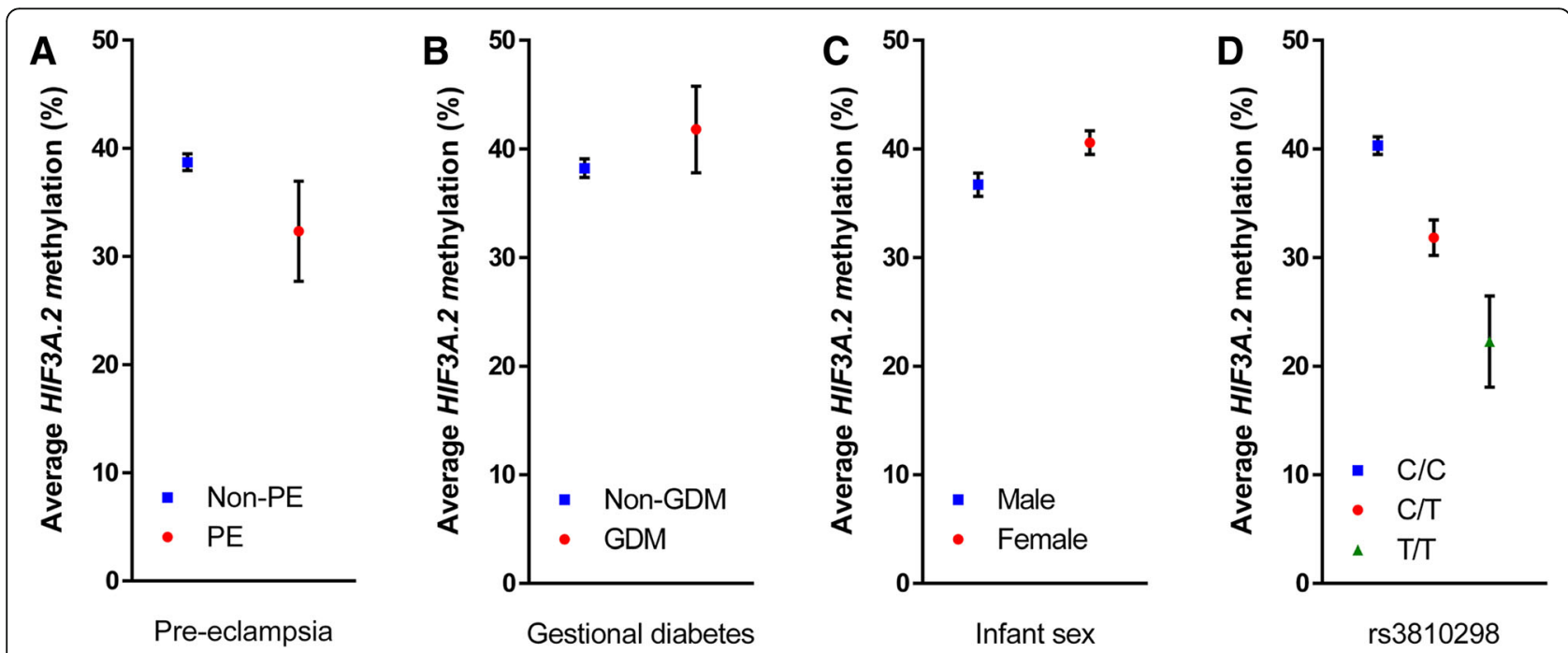

Fig. 2 Distribution of average HIF3A.2 methylation stratified by a pre-eclampsia (PE), b gestational diabetes (GDM), c infant sex and $\mathbf{d}$ rs 3810298 genotype. Error bars are mean $\pm 95 \%$ confidence interval 
Table 3 Average methylation of HIF3A.1 and HIF3A.2 by genotype for each of the 14 tag SNPs considered in this analysis

\begin{tabular}{|c|c|c|c|c|c|c|}
\hline \multirow{2}{*}{$\frac{\text { SNP }}{\text { rs62111812 }}$} & \multicolumn{3}{|c|}{ HIF3A.1 methylation $(n=410)$} & \multicolumn{3}{|c|}{ HIF3A.2 methylation $(n=595)$} \\
\hline & Mean (SD) & $n$ & $p$ & Mean (SD) & $n$ & $p$ \\
\hline $\mathrm{G} / \mathrm{G}$ & $70.02(4.59)$ & 313 & 0.22 & $37.95(9.96)$ & 438 & 0.1 \\
\hline $\mathrm{G} / \mathrm{A}$ & $70.93(4.74)$ & 89 & & $39.93(8.74)$ & 144 & \\
\hline $\mathrm{A} / \mathrm{A}$ & $71.19(1.71)$ & 8 & & $39.47(10.37)$ & 13 & \\
\hline \multicolumn{7}{|l|}{ rs112087991 } \\
\hline $\mathrm{T} / \mathrm{T}$ & $70.39(4.47)$ & 359 & 0.22 & $39.46(9.44)$ & 522 & $<0.0001$ \\
\hline $\mathrm{T} / \mathrm{C}$ & $69.18(5.38)$ & 49 & & $31.54(8.67)$ & 70 & \\
\hline $\mathrm{C} / \mathrm{C}$ & $69.57(0.61)$ & 2 & & $27.17(8.30)$ & 3 & \\
\hline \multicolumn{7}{|l|}{ rs8102595 } \\
\hline $\mathrm{A} / \mathrm{A}$ & $69.97(4.60)$ & 331 & 0.04 & $38.63(9.83)$ & 477 & 0.7 \\
\hline$A / G$ & $71.37(4.46)$ & 76 & & $37.79(9.28)$ & 115 & \\
\hline $\mathrm{G} / \mathrm{G}$ & $72.07(2.20)$ & 3 & & $37.56(9.56)$ & 3 & \\
\hline \multicolumn{7}{|l|}{ rs3826795 } \\
\hline $\mathrm{C} / \mathrm{C}$ & $70.65(4.18)$ & 250 & 0.07 & $40.42(9.14)$ & 364 & $<0.0001$ \\
\hline$C / T$ & $69.68(5.23)$ & 142 & & $35.76(9.45)$ & 196 & \\
\hline $\mathrm{T} / \mathrm{T}$ & $69.03(4.16)$ & 18 & & $33.28(11.52)$ & 35 & \\
\hline \multicolumn{7}{|l|}{ rs3810298 } \\
\hline$C / C$ & $70.42(4.27)$ & 337 & 0.26 & $40.32(9.02)$ & 476 & $<0.0001$ \\
\hline $\mathrm{C} / \mathrm{T}$ & $69.44(5.95)$ & 67 & & $31.84(8.63)$ & 109 & \\
\hline $\mathrm{T} / \mathrm{T}$ & $69.44(4.36)$ & 6 & & $22.26(5.88)$ & 10 & \\
\hline \multicolumn{7}{|l|}{ rs140454328 } \\
\hline $\mathrm{T} / \mathrm{T}$ & $70.26(4.61)$ & 398 & 0.71 & $38.49(9.72)$ & 563 & 0.87 \\
\hline $\mathrm{T} / \mathrm{C}$ & $69.75(4.01)$ & 12 & & $38.08(9.90)$ & 31 & \\
\hline $\mathrm{C} / \mathrm{C}$ & - & 0 & & $33.83(0)$ & 1 & \\
\hline \multicolumn{7}{|l|}{ rs36063219 } \\
\hline$C / C$ & $70.23(4.63)$ & 393 & 0.78 & $38.41(9.76)$ & 576 & 0.46 \\
\hline$C / T$ & $70.55(3.80)$ & 17 & & $40.08(8.39)$ & 19 & \\
\hline $\mathrm{T} / \mathrm{T}$ & - & 0 & & - & 0 & \\
\hline \multicolumn{7}{|l|}{ rs3752207 } \\
\hline$C / C$ & $70.23(4.43)$ & 357 & 0.21 & $38.31(9.54)$ & 516 & 0.29 \\
\hline$C / A$ & $70.66(4.95)$ & 48 & & $39.06(11.02)$ & 72 & \\
\hline $\mathrm{A} / \mathrm{A}$ & $66.83(10.11)$ & 5 & & $43.75(7.81)$ & 7 & \\
\hline \multicolumn{7}{|l|}{ rs4803929 } \\
\hline$C / C$ & $70.13(4.64)$ & 355 & 0.04 & 38.6 (9.78) & 510 & 0.29 \\
\hline $\mathrm{C} / \mathrm{T}$ & 70.7 (4.01) & 53 & & $37.41(9.25)$ & 83 & \\
\hline $\mathrm{T} / \mathrm{T}$ & $77.88(4.77)$ & 2 & & $46.49(8.69)$ & 2 & \\
\hline \multicolumn{7}{|l|}{ rs9304657 } \\
\hline $\mathrm{T} / \mathrm{T}$ & $70.34(4.00)$ & 181 & 0.75 & $38.31(10.18)$ & 288 & 0.92 \\
\hline $\mathrm{T} / \mathrm{C}$ & $70.27(5.00)$ & 178 & & $38.58(9.48)$ & 237 & \\
\hline$C / C$ & $69.80(5.12)$ & 51 & & $38.72(8.58)$ & 70 & \\
\hline \multicolumn{7}{|l|}{ rs76789866 } \\
\hline $\mathrm{T} / \mathrm{T}$ & $70.26(4.58)$ & 385 & 0.82 & $38.83(9.61)$ & 546 & 0.01 \\
\hline $\mathrm{T} / \mathrm{C}$ & $70.04(4.79)$ & 25 & & $34.29(9.85)$ & 47 & \\
\hline $\mathrm{C} / \mathrm{C}$ & - & 0 & & $37.43(17.94)$ & 2 & \\
\hline
\end{tabular}


Table 3 Average methylation of HIF3A.1 and HIF3A.2 by genotype for each of the 14 tag SNPs considered in this analysis (Continued)

\begin{tabular}{|c|c|c|c|c|c|c|}
\hline \multirow{2}{*}{$\frac{S N P}{\text { rs7595265 }}$} & \multicolumn{3}{|c|}{ HIF3A.1 methylation $(n=410)$} & \multicolumn{3}{|c|}{ HIF3A.2 methylation $(n=595)$} \\
\hline & & & & & & \\
\hline $\mathrm{T} / \mathrm{T}$ & $70.28(4.67)$ & 382 & \multirow[t]{3}{*}{0.54} & $38.32(9.70)$ & 558 & \multirow[t]{3}{*}{0.15} \\
\hline T/A & $69.73(3.29)$ & 28 & & $40.71(9.81)$ & 37 & \\
\hline $\mathrm{A} / \mathrm{A}$ & - & 0 & & - & 0 & \\
\hline \multicolumn{7}{|c|}{ rs917946 } \\
\hline $\mathrm{A} / \mathrm{A}$ & $70.25(4.58)$ & 328 & \multirow[t]{3}{*}{0.96} & $38.07(9.51)$ & 477 & \multirow[t]{3}{*}{0.05} \\
\hline$A / G$ & $70.23(4.72)$ & 79 & & $39.74(10.47)$ & 111 & \\
\hline $\mathrm{G} / \mathrm{G}$ & $69.44(2.17)$ & 3 & & $45.28(7.40)$ & 7 & \\
\hline \multicolumn{7}{|c|}{ rs12459580 } \\
\hline $\mathrm{G} / \mathrm{G}$ & $70.08(4.19)$ & 119 & \multirow[t]{3}{*}{0.65} & $38.71(8.36)$ & 168 & \multirow[t]{3}{*}{0.93} \\
\hline $\mathrm{G} / \mathrm{C}$ & $70.44(4.74)$ & 212 & & $38.36(9.89)$ & 295 & \\
\hline $\mathrm{C} / \mathrm{C}$ & $69.95(4.77)$ & 79 & & $38.39(10.91)$ & 132 & \\
\hline
\end{tabular}

$p$ value is from one-way ANOVA. SD standard deviation

per percentage increase in granulocytes, 95\% CI 0.02, $0.20, p=0.01 ;-0.14 \%$ decrease per percentage increase in lymphocytes, $95 \%$ CI $-0.23,-0.05, p=0.002$ ). Due to collinearity between granulocyte and lymphocyte proportions, the final model was adjusted for only lymphocyte proportion, which modestly decreased the association between gestational age and average HIF3A.2 methylation $(0.99 \%$ increased methylation per week, 95\% CI $0.41,1.57, p=0.001$ ), with no effect on other associations tested. We also examined both GDM and pre-eclampsia as a composite exposure. Methylation associated with both outcomes as mutually exclusive factors, with the GDM-only group having 4.87\% higher average methylation than the non-GDM, nonpre-eclampsia group, and the pre-eclampsia-only group having 5.98\% lower average methylation.

Table 4 Final linear regression model adjusting for all key variables with average HIF3A.2 methylation as outcome

\begin{tabular}{llll}
\hline & \multicolumn{4}{l}{ Average across HIF3A.2 $(n=513)$} \\
\cline { 2 - 4 } & $\beta(\mathrm{SE})$ & $p$ & $95 \% \mathrm{Cl}$ \\
\hline GDM & $4.62(1.77)$ & 0.009 & 1.14 to 8.09 \\
Pre-eclampsia & $-5.20(2.19)$ & 0.02 & -9.51 to -0.89 \\
Sex (male) & $-3.77(0.76)$ & $<0.001$ & -5.26 to -2.29 \\
Gestational age (weeks) & $1.21(0.27)$ & $<0.001$ & 0.68 to 1.74 \\
rs3810298 & & & \\
\multicolumn{1}{c}{ C/T } & $-8.31(0.97)$ & $<0.001$ & -10.22 to -6.41 \\
T/T & $-16.91(2.73)$ & $<0.001$ & -22.27 to -11.54
\end{tabular}

Effect sizes ( $\beta$ ) given as percentage methylation. $\beta$ for rs3810298 categories is the difference from homozygote major allele (C/C). GDM gestational diabetes, SE standard error, $\mathrm{Cl}$ confidence interval. No other SNPs were strongly associated with HIF3A.2 methylation after adjustment for rs3810298, and a linear regression model with just rs 3810298 accounted for a similar amount of variation in HIF3A.2 methylation measures and the model with six SNPs $\left(R^{2}\right.$ for minimal model $=0.162, R^{2}$ for full model $=0.177, p=0.29$ )
There was no evidence for interaction effects between infant sex or genotype with other key measures in terms of their effects on average HIF3A.2 methylation. In the unit-specific analysis, pre-pregnancy BMI was associated with CpG11 methylation $(r=0.08, p=0.04)$ (Additional file 5), but adjusting for maternal pre-pregnancy BMI did not substantially alter the estimate of effects. Similarly, the inclusion of interaction effects or prepregnancy BMI did not substantially improve the fit of the linear regression model. The final model applied to site-specific HIF3A.2 methylation is shown in Additional file 6 . We focussed on the average methylation only as no specific CpG site(s) showed evidence of a differential degree of association.

In CpG unit-specific analysis for HIF3A.1, gestational age was positively associated with methylation at CpG5 specifically $(r=0.12, p=0.007)$, while maternal age at conception was weakly, positively associated with CpG5 methylation $(r=0.08, p=0.07)$ (Additional file 7). Adjusting for these measures did not alter the association between infant sex and average HIF3A.1 methylation. Of the cell types considered in the sensitivity analysis, activated $\mathrm{T}_{\text {reg }}$ cells showed the strongest evidence of an association with average HIF3A.1 methylation (-1.24\% decreased methylation per activated $\mathrm{T}_{\text {reg }}$ percentage, $95 \% \mathrm{CI}-2.50$ to $0.03, p=0.06$ ), but adjusting for cellular proportions did not alter any of the observed associations between HIF3A.1 methylation and maternal and infant factors.

\section{Discussion}

We found evidence for independent association of HIF3A methylation at birth with important pregnancyrelated outcomes, offspring anthropometric measures and genetic variation, particularly around HIF3A.2, previously linked to maternal BMI [19]. This is consistent 


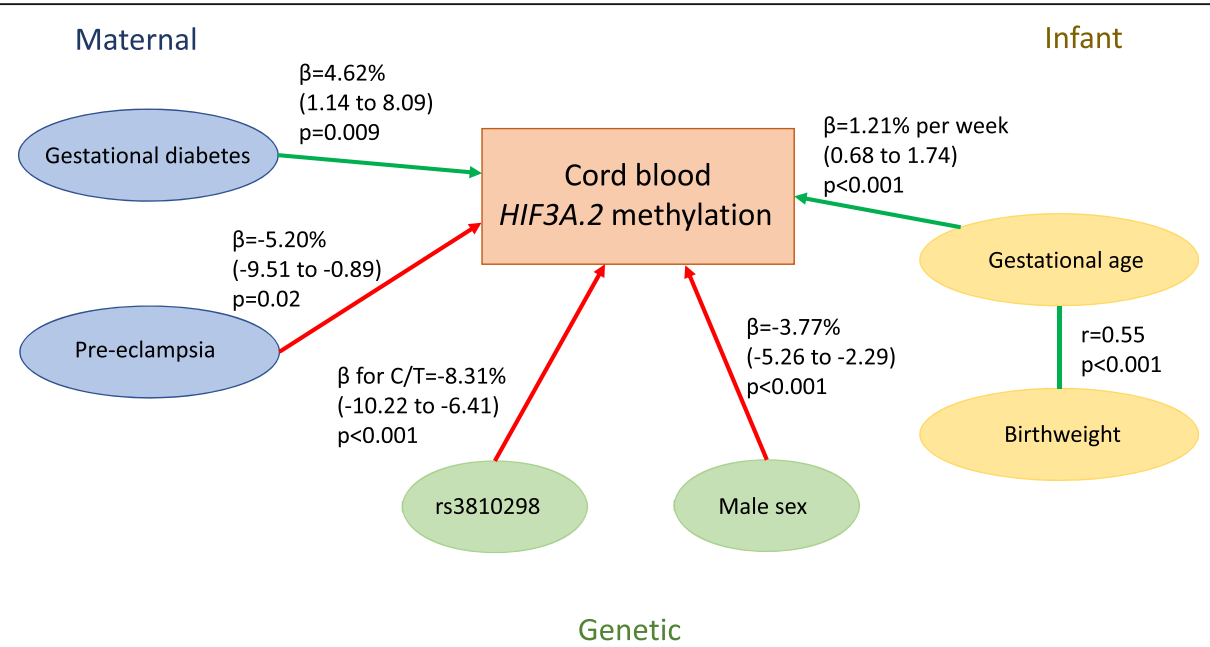

Fig. 3 Diagram of associations between maternal, infant and genetic factors and HIF3A.2 methylation in cord blood. Green arrows denote positive associations and red arrows denote negative associations. Beta values (and 95\% confidence interval in brackets) and $p$ values are from the final linear regression model for HIF3A.2 methylation (Table 4)

with multiple biological pathways intersecting through HIF3A epigenetic variation. In this study, our observed cord blood methylation levels were comparable with a previous cord blood study using genome-wide $\mathrm{CpG}$ probes across HIF3A.1 and HIF3A.2 [22], with HIF3A.1 hypermethylated compared to HIF3A.2, which had intermediate levels of methylation. Future studies should investigate the nature of this intermediate methylation to determine whether it may be due in part to monoallelic methylation or cellular heterogeneity. Distinct environmental effects on methylation at the two regions is consistent with a previous longitudinal childhood cohort study [19] that reported a positive association between maternal pre-pregnancy BMI and cord blood methylation at CpG probes in and near HIF3A.2 but not HIF3A.1, and reported a weak negative correlation in methylation between the two regions, as we have found here.

\section{HIF-3a regulation and function}

The HIF3A gene produces up to eight different transcripts from potentially three different promoter regions [23], with the likelihood that HIF3A.2 methylation influences expression of a subset. Hypoxia has been reported to upregulate transcripts associated with all 3 promoter regions [23]. It has previously been found the DNA methylation of HIF3A.1 CpG5 is negatively associated with expression in adipose tissue [6], though a later study reported that BMI, but not methylation, is negatively associated with expression in adipose tissue [9]. The decreased methylation of HIF3A.2 in response to pre-eclampsia might be anticipated to increase gene expression, which is consistent with upregulation of HIF3A transcription in response to hypoxia. A genome-wide study investigating associations between gene expression measured by RNA sequencing and
DNA methylation [24] did not find any associations between any of the four CpG probes analysed (cg27146050, cg22891070, cg16672562 and cg26749414) in fibroblasts, $\mathrm{T}$ cells and a lymphoblastoid cell line. However, differential HIF3A.2 methylation may influence a specific subset of HIF $3 A$ isoforms rather than overall gene expression.

\section{A role in pre-eclampsia?}

To our knowledge, this is the first study to report evidence for a link between pre-eclampsia and HIF3A methylation (Additional file 1). Pre-eclampsia is characterised by inappropriate spiral artery development during placentation [25], often in association with the development of a hypoxic environment for the developing foetus. As such, the differential HIF3A.2 methylation we have observed may reflect an adaptation or response of the hypoxia response pathway to a suboptimal environment. Though the potential role of HIF3A in pre-eclampsia has not yet been elucidated, it has been established that the broader HIF family is involved with high levels of HIF1A expression in the placenta [26], and it has been proposed that dysregulated HIF1A may contribute to the genesis of pre-eclampsia through altered placental development.

\section{Impact of gestational diabetes}

Previous studies have reported an association between GDM and HIF3A methylation [22], though this was in the area covered by HIF3A.1. While we have found novel evidence for GDM influencing average methylation in HIF3A.2, no such association was observed for HIF3A.1. However, there were relatively few women in our population-derived cohort with GDM ( $n=17$ for the HIF3A.1 sub-cohort) compared to the previous study 
that was enriched for mothers with GDM $(n=68)$ [22]. As such, we were powered for identifying larger effects in HIF3A.1 (80\% power for a $3.37 \%$ increase in methylation) than the small magnitude of association previously reported (a 1.3\% increase in univariate analysis, for which we had $19.2 \%$ power). HIF3A plays a role in glucose metabolism [27] and induces adipocyte-related gene expression [28] so may be involved in mediating the effects of maternal GDM on the infant's development.

\section{Impact of offspring sex}

Previous studies have considered infant sex as a covariate to control for in multivariable analysis (Additional file 1 ) but have focussed primarily on $\mathrm{CpG}$ sites within HIF3A.1. None have found an effect of sex on methylation. This is consistent with the modest association observed between infant sex and HIF3A.1 methylation in this study. Interestingly however, we found a strong association between male sex and lower HIF3A.2 methylation. There is little evidence in the existing literature for sex-specific differences in the function of HIF $3 A$ in adulthood; however, a mouse study reported that HIF3A expression increased in the placenta in response to maternal stress during pregnancy for placentas of male offspring but not females [29]. More broadly, there is well-established evidence for sexual dimorphism of the placenta, with reported differences in gene expression, adaptation to in utero exposures and pregnancy outcomes between the sexes [30,31]. Sexual dimorphism may be driving the average HIF3A.2 methylation differences between sexes we have observed in this study through the differential placental regulation of growth and availability of oxygen.

\section{Growth and adiposity}

We did not find strong evidence of an association between infant adiposity or weight with average HIF3A.1 methylation. Previous studies have primarily investigated methylation of three CpG sites in HIF3A.1, CpG1.2, CpG5 and CpG 6.7.8, and reported associations between methylation and adiposity or BMI in later childhood and adult timepoints $[4,6,8,9,20,21]$, particularly in adipose tissue but also whole blood. These studies have all used BMI as a measure of adiposity, often in combination with other measures, but BMI is not a suitable measure for adiposity at birth and instead triceps and subscapular skinfold thickness was used for our study. A positive association between methylation of these three key CpG sites and subscapular skinfold thickness has been reported in umbilical cord tissue in a previous birth cohort study [17], but we did not find evidence for associations between methylation of these three key CpG sites and growth or adiposity in site-specific analysis in cord blood (Additional file 7), potentially indicating a tissue-specific effect. A similar lack of evidence for associations was previously reported by Richmond et al. using comparable anthropometry and cord blood $(n=1018)$ [19]. However, contrasting with this previous study, which also considered BMI on a continuous scale, we did not find evidence of maternal pre-pregnancy BMI associating with average HIF3A.2 methylation $(n=518)$. The CpG site previously found to be most strongly associated with prepregnancy BMI was HIF3A.2 CpG13, but we found no evidence of an association between maternal pre-pregnancy BMI and methylation of CpG13 (Additional file 5).

\section{Impact of offspring HIF3A genotype}

The impact of genetic variation on DNA methylation is pervasive [24, 32-38] but only partly understood [39]. In the current study, we captured genetic variation in an 83-kb region of HIF3A and upstream and downstream SNPs. There were 291 common (minor allele frequency $>0.01$ ) SNPs in this region, none of which were in the same location as $\mathrm{CpG}$ sites we measured, though there were four of these SNPs within HIF3A.1 or HIF3A.2. Previous studies have reported associations of rs 8102595 and rs3826795 with methylation of CpG sites within HIF3A.1 in adult tissues $[6,8]$ and umbilical cord tissue [17], and we found modest evidence for these SNPs influencing average HIF3A.1 methylation, though rs3826795 was more strongly associated with average HIF3A. 2 methylation. Of the 14 tag SNPs considered in this analysis, rs3810298 showed the greatest effect on average HIF3A.2 methylation (Table 3 ) and is situated $6488 \mathrm{bp}$ upstream from the start of HIF3A.2 (Additional file 8). The other two SNPs that showed a strong association with average HIF3A.2 methylation, rs112087991 and rs3826795, had a high level of linkage disequilibrium with rs3810298 (Additional file 3) indicating there may be a single SNP driving this effect on average methylation in HIF3A.2. The rs3826795 SNP was used as a tag for a total of 14 common SNPs, and of these, three (rs2072491, rs4802306 and rs3810298) are located in the second intron of HIF $3 A$ and positioned in regions of open chromatin that have been identified in multiple blood cell lines as part of the ENCODE project [40]. As such, the genotype of these SNPs may impact protein binding in these regions, and subsequent regulation of HIF $3 A$, but this has yet to be tested. To our knowledge, we are the first to report associations between HIF3A genetic variation and infant cord blood methylation within the HIF3A.2 region.

The key strength of this study is the combination of pregnancy health measures, infant anthropometry, genetic variation data and relatively large sample size in a population-based cohort. We have also measured locusspecific methylation allowing us to look at average methylation across two HIF3A regions as well as key 
CpG sites. The key limitation is the missing data across covariates and CpG units. As such our sample size for some analyses is smaller than some previous studies, particularly for the HIF3A.1 region, limiting our power to detect small effect sizes (Additional file 1). We did not correct for multiple testing across the ten maternal and infant measures considered in association with HIF3A.1 and HIF3A.2 methylation. Thus, there is a possibility of false positive associations within our findings. However, these regions and measures were not agnostically chosen but based on prior literature. Further, these associations show strong evidence of persisting in multivariable linear regression modelling and were largely unchanged by the various sensitivity analyses performed. We lack information regarding participant interventions to treat pre-eclampsia or GDM that may impact findings. The functional consequences of the observed methylation variation at a specific promoter of HIF3A remain unclear, particularly given the lack of publicly available data on the expression and regulation of the different isoforms of this gene. Previous studies have examined total expression only, rather than isoformspecific expression, or have focussed solely on as on methylation at one of two HIF3A promoters that likely regulate these different isoforms $[6,9]$. Future functional studies are clearly warranted in this regard to test the influence of methylation on the regulation of different isoforms of this gene. An additional consideration is that we have measured methylation in cord blood, and due to the tissue-specific nature of DNA methylation, the importance of the differential methylation we observed in this study on other tissues relevant to HIF3A is unclear, particularly as $H I F 3 A$ transcription variants are differentially expressed between tissues [41].

\section{Conclusions}

Several early-life and genetic factors appear to be associated with differential cord blood HIF3A DNA methylation at birth, though the potential impact of altered HIF3A methylation on gene expression, health and development from early childhood has yet to be wellcharacterised. Given the association between GDM and pre-eclampsia and both adverse maternal and infant health outcomes in later life, further studies are required to investigate the persistence of HIF3A methylation patterns beyond early infancy and their relevance to subsequent health outcomes.

\section{Methods}

\section{Participant recruitment and follow-up}

The Barwon Infant Study [42] (BIS) is an Australian birth cohort consisting of 1074 mother-infant dyads, with the aim of investigating early-life development and disease across several domains, including immune development, cardiovascular health, neurodevelopment and respiratory health. Women between 15 and 32 completed weeks of pregnancy were recruited from two hospitals but were excluded if they (1) were no longer residents in the Barwon region at the time of their child's birth, (2) were younger than 18 years at 28 weeks of pregnancy, (3) were without Australian citizenship or permanent residency to allow for follow-up, (4) were unable to complete questionnaires or provide informed consent, (5) had a previous child in the BIS cohort (excluding twins) or (6) were planning to store their child's cord blood privately. Neonates were excluded if they (1) had a gestational age less than 32 weeks, (2) were diagnosed with a serious illness or (3) had a genetic disease or congenital malformation. Over the 3-year recruitment period, 3933 women were contacted for recruitment and 1158 were recruited during pregnancy. After birth, 1074 mother-infant dyads remained eligible.

\section{Prenatal and infant measures}

Health information was obtained from medical records and standardised clinical information. Gestational diabetes was defined as plasma glucose greater than 5.1 $\mathrm{mmol} / \mathrm{L}$ for fasting or greater than $8.5 \mathrm{mmol} / \mathrm{L} 2 \mathrm{~h}$ after 75-g oral glucose load [43]. Pre-eclampsia was defined as per International Society for the Study of Hypertension in Pregnancy (ISSHP) criteria, with onset of high blood pressure $(>140 / 90)$ and proteinuria $(>0.3 \mathrm{~g} / 24$ hr) after 20 weeks of gestation [44]. Maternal prepregnancy BMI was calculated from self-reported weight and height measures.

Infant sex, gestational age, birth weight and anthropometry were obtained within two days of birth. Zscores adjusted for sex and age were calculated for birth weight based on the revised British UK-WHO growth charts [45]. Triceps and subscapular skinfold thickness were measured using Holtain callipers. Sum of skinfolds (triceps and subscapular) was used as a proxy measure for central adiposity. The coefficient of reliability for newborn skinfold measures range from 75 to $93 \%$ [46].

\section{DNA extraction and HIF3A methylation}

Genomic DNA was extracted from whole cord blood using the QIAamp DNA QIAcube HT Kit (QIAGEN, Hilden, Germany), following manufacturer's instructions. Bisulphite conversion was performed using the MagPrep Lightning Conversion Kit (Zymo Research, Irvine, CA, USA). Assays for methylation of two promoter regions of HIF3A (HIF3A.1 and HIF3A.2; hg38:chr19:46,298, 243-46,298,580 and hg38:chr19:46,303,864-46,304,196, respectively) were designed using EpiDesigner (Agena Bioscience, San Diego, CA, USA). Details are shown in Additional file 8 and Additional file 9. DNA methylation 
and quality control were quantified as previously described [47, 48], using the SEQUENOM MassARRAY EpiTYPER platform. Methylation level was determined as the average proportion of methylation within CpG units, with each $\mathrm{CpG}$ unit containing one to four $\mathrm{CpG}$ sites. The HIF3A.1 assay covered sites previously linked to adult and childhood BMI and adiposity [4, 7-9, 17-21] and measured six CpG units containing a total of $11 \mathrm{CpG}$ sites. The HIF3A.2 assay covered a site recently associated with maternal BMI [19] and measured 13 CpG units containing a total of 20 CpG sites. HIF3A.1 was measured in a subsample of 490 infants, with complete methylation data for all six CpG units available for 423 infants. HIF3A.2 methylation was subsequently measured in the full cohort of 938 infants, with complete methylation data for all $13 \mathrm{CpG}$ units available for 609 infants.

To account for potential contribution from the cellular heterogeneity of the cord blood samples, populations of granulocytes, monocytes and lymphocytes were assessed by flow cytometry (FACsCalibur) and presented as a percentage of total white blood cells $(n=938)$ [49]. This was used in sensitivity analyses. In addition, proportions of naïve and activated $\mathrm{T}_{\text {reg }}$ cells (as a percentage of total CD4+ T-cells) were available for a subset of infants $(n=464)$ and were also considered in sensitivity analyses.

\section{HIF3A genotyping}

Genotypes were measured using the Infinium Global Screening Array-24 v1.0 BeadChip (Illumina, San Diego, CA, USA). The Sanger Imputation Service (Wellcome Sanger Institute, Hinxton, UK) was used for imputing SNPs not captured in the initial genotyping using the EAGLE2+PBWT phasing and imputation pipeline with the Haplotype Reference Consortium reference panel [50]. The imputed SNPs were filtered for an information score greater than 0.8. The common SNPs (minor allele frequency of at least 0.01 ) were extracted for analysis (hg38: chr19:46,278,743-46,361,743), resulting in 291 SNPs. Due to the large number of SNPs, Haploview (Broad Institute, Cambridge, MA, USA) [51] was used to identify 14 tag SNPs using a $r^{2}$ threshold of 0.1 and mandating the inclusion of two SNPs of interest identified previously [17] (rs8102595 and rs3826795).

\section{Statistical analysis}

As methylation within a region was generally highly correlated, we considered the average methylation. Covariates of interest were identified with univariate statistical testing (unpaired two-tailed Student's $t$ test, correlation coefficient test or ANOVA as appropriate) of the average methylation for each region. The degree of correlation of tag SNPs associated with methylation was used to identify a single SNP as a proxy for the cluster of linked SNPs (based on Chi-squared tests) (Additional file 3).
Linear regression models with methylation as the outcome were first built with by identifying factors associated with methylation, and then once the core model was determined, possible interaction effects between infant sex, genetic variation, and other measures on methylation were added to the model and evaluated sequentially. Participants were only included in multivariable models if they had complete data for all included variables. Subsequent analysis of specific CpG unit methylation was performed to identify secondary maternal or infant measures to be included in sensitivity analysis. Other covariates added separately to the linear regression model for sensitivity analysis included proportions of various white blood cells in the cord blood samples and technical variables (bisulphite conversion batch and methylation quantification batch). Analyses including blood cell proportions were also adjusted for infant exposure to labour prior to delivery (any or none). The covariates examined in sensitivity analysis were considered to impact on the model if they altered the beta value of the main effects by $10 \%$ or more. The likelihood ratio test was used to compare nested linear regression models to evaluate if additional terms in the model were useful for explaining additional outcome variance. Stata 15 IC (StataCorp, College Station, TX, USA) was used for analysis.

\section{Additional files}

Additional file 1: Summary table of studies investigating HIF3A DNA methylation in the context of obesity, adiposity, or early-life influences. (DOCX $21 \mathrm{~kb}$ )

Additional file 2: Tables of the pairwise associations between the HIF3A.1 and HIF3A.2 CpG units measured in this study. The first spreadsheet in the file gives the $r$ values, the second spreadsheet gives the $p$ values. (XLSX $18 \mathrm{~kb}$ )

Additional file 3: Visualisation of linkage disequilibrium between the 14 tag SNPs considered in this analysis from Haploview. The numbers in individual pairwise boxes are the D', a measure of linkage disequilibrium. A stronger red colour for a box indicates the two SNPs are more strongly linked. The white bar at the top indicates the relative genomic position of each SNP. (DOCX $50 \mathrm{~kb}$ )

Additional file 4: Summary tables of the univariate tests between rs3810298 genotype and (a) pre-eclampsia, (b) gestational diabetes, (c) birth weight, and (d) gestational age. (XLSX $11 \mathrm{~kb}$ )

Additional file 5: Table of associations between cohort characteristics and methylation of unit-specific HIF3A.2 CpG methylation. (DOCX 23 kb)

Additional file 6: Table of final linear regression model adjusting for all key variables with unit-specific HIF3A.2 methylation as outcome, applied to all measured HIF3A.2 CpG units. (DOCX $20 \mathrm{~kb}$ )

Additional file 7: Table of associations between cohort characteristics and methylation of unit-specific HIF3A.1 CpG methylation. (DOCX 18 kb)

Additional file 8: Annotated UCSC genome browser (http://genome. ucsc.edu) view of the HIF3A gene region and Epityper assays. (a) HIF3A gene on chromosome 19. Gene transcription is from left to right. Multiple splice variants are shown, with solid dark segments indicating exons, and the connecting lines indicating introns. The positions of SNPS included in this analysis are shown in green and labelled. (b) The HIF3A.1 region, with CPG sites in red. (c) The HIF3A.2 region, with CPG sites in 
red. The measurable CpG sites are numbered based on the predicted cleavage pattern from the Epityper in silico prediction. CpG units that contain CpG sites of interest from previous literature have the CpG site reference in brackets beneath the number. (DOCX $111 \mathrm{~kb}$ )

Additional file 9: Table of primer and assay information for HIF3A.1 and HIF3A.2. (DOCX $13 \mathrm{~kb})$

\section{Abbreviations}

A: Adenine; ANOVA: Analysis of variance; BIS: Barwon Infant Study; BMl: Body mass index; C: Cytosine; Cl: Confidence interval; CpG: Cytosineguanine dinucleotide; DNA: Deoxyribonucleic acid; DOHaD: Developmental Origins of Health and Disease; EWAS: Epigenome-wide association study; G: Guanine; GDM: Gestational diabetes mellitus; hg38: Genome Reference Consortium human genome build 38; HIF3A: Hypoxia-inducible factor $3 a$ gene; HIF-3a: Hypoxia-inducible factor 3a; ISSHP: International Society for the Study of Hypertension in Pregnancy; PBWT: Positional Burrows-Wheeler transform; SD: Standard deviation; SE: Standard error; SNP: Single nucleotide polymorphism; T: Thymine; UK-WHO: United Kingdom-World Health Organisation

\section{Acknowledgements}

We thank the BIS participants for the generous contribution they have made to this project. We also thank current and past staff for their efforts in recruiting and maintaining the cohort and in obtaining and processing the data and biospecimens. We thank QIMR Berghofer Medical Research Institute and the Erasmus MC University Medical Center for their role in coordinating and performing the genotyping of BIS samples.

The members of the Barwon Infant Study Investigator Team are the following: Peter Vuillermin, Barwon Health, Deakin University, the Murdoch Children's Research Institute, and University of Melbourne; Anne-Louise Ponsonby, John Carlin, Katie Allen, Mimi Tang, Richard Saffery, Sarath Ranganathan, and David Burgner, the Murdoch Children's Research Institute, University of Melbourne; Terry Dwyer, the Murdoch Children's Research Institute and the George Institute for Global Health; Kim Jachno, the Murdoch Children's Research Institute; and Peter Sly, University of Queensland, Queensland Children's Medical Research Institute.

\section{Authors' contributions}

TM undertook DNA extraction and methylation measurement in samples and analysis and writing of the manuscript. ALP was a chief investigator for the Barwon Infant Study and a major contributor to analysis and interpretation of data. VJ undertook DNA extraction and methylation measurement in samples. FC co-ordinated blood collection and anthropometry measurement of participants and undertook cell sorting of blood samples. DB was a major contributor to planning of anthropometry collection and interpretation of data. PV was a chief investigator for the Barwon Infant Study. JR was a major contributor to analysis and interpretation of data. RS was a major contributor to coordination of blood collection, DNA extraction and methylation measurement; analysis and interpretation of data; and writing. All authors assisted in critical revision of the manuscript and approved the final version.

\section{Funding}

The establishment work and infrastructure for the BIS was provided by the Murdoch Children's Research Institute, Deakin University and Barwon Health. Subsequent funding was secured from the National Health and Medical Research Council of Australia, The Jack Brockhoff Foundation, the Scobie Trust, the Shane O'Brien Memorial Asthma Foundation, the Our Women's Our Children's Fund Raising Committee Barwon Health, The Shepherd Foundation, the Rotary Club of Geelong, the Ilhan Food Allergy Foundation, GMHBA Limited and the Percy Baxter Charitable Trust, Perpetual Trustees. Inkind support was provided by the Cotton On Foundation and CreativeForce. The study sponsors were not involved in the collection, analysis and interpretation of data; writing of the report; or the decision to submit the report for publication. Research at Murdoch Children's Research Institute is supported by the Victorian Government's Operational Infrastructure Support Program. This work was also supported by a Research Training Program Stipend through University of Melbourne (to TM), NHMRC Senior Research Fellowships (APP1008396 to ALP; APP1045161 to RS); and an NHMRC Dementia Research Leader Fellowship (APP1135727 to JR).

\section{Availability of data and materials}

The datasets used and/or analysed during the current study are available from the corresponding author on reasonable request.

\section{Ethics approval and consent to participate}

The protocol for this study was approved by the Barwon Health Human Research Ethics Committee. Participants provided written informed consent prior to recruitment.

\section{Consent for publication}

Not applicable.

\section{Competing interests}

The authors declare that they have no competing interests.

\section{Author details}

${ }^{1}$ Murdoch Children's Research Institute, Parkville, Australia. ${ }^{2}$ Department of Paediatrics, University of Melbourne, Parkville, Australia. ${ }^{3}$ The Florey Institute of Neuroscience and Mental Health, Parkville, Australia. ${ }^{4}$ School of Medicine, Deakin University, Geelong, Australia. ${ }^{5}$ Child Health Research Unit, Barwon Health, Geelong, Australia. 'Department of Paediatrics, Monash University, Clayton, Australia. ${ }^{7}$ School of Public Health \& Preventive Medicine, Monash University, Melbourne, Australia.

Received: 26 November 2018 Accepted: 27 May 2019

Published online: 01 July 2019

\section{References}

1. Gluckman PD, Hanson MA. The developmental origins of the metabolic syndrome. Trends Endocrinol Metab. 2004;15(4):183-7.

2. Novakovic B, Saffery R. The importance of the intrauterine environment in shaping the human neonatal epigenome. Epigenomics. 2013;5(1):1-4.

3. Barker DJP. The origins of the developmental origins theory. J Intern Med. 2007;261(5):412-7.

4. Demerath EW, Guan W, Grove ML, Aslibekyan S, Mendelson M, Zhou YH, et al. Epigenome-wide association study (EWAS) of BMI, BMI change and waist circumference in African American adults identifies multiple replicated loci. Hum Mol Genet. 2015;24(15):4464-79.

5. Jelenkovic A, Yokoyama Y, Sund R, Honda C, Bogl LH, Aaltonen S, et al. Zygosity differences in height and body mass index of twins from infancy to old age: a study of the CODATwins project. Twin Res Hum Genet. 2015; 18(5):557-70.

6. Dick KJ, Nelson CP, Tsaprouni L, Sandling JK, Aissi D, Wahl S, et al. DNA methylation and body-mass index: a genome-wide analysis. Lancet (London, England). 2014;383(9933):1990-8.

7. Agha G, Houseman EA, Kelsey KT, Eaton CB, Buka SL, Loucks EB. Adiposity is associated with DNA methylation profile in adipose tissue. Int J Epidemiol. 2015:44(4):1277-87.

8. Pfeiffer S, Krüger J, Maierhofer A, Böttcher Y, Klöting N, El Hajj N, et al. Hypoxia-inducible factor $3 \mathrm{~A}$ gene expression and methylation in adipose tissue is related to adipose tissue dysfunction. Sci Rep. 2016;6:27969.

9. Main AM, Gillberg L, Jacobsen AL, Nilsson E, Gjesing AP, Hansen T, et al. DNA methylation and gene expression of HIF3A: cross-tissue validation and associations with BMI and insulin resistance. Clin Epigenetics. 2016;8:89.

10. Huang Y-T, Chu S, Loucks EB, Lin C-L, Eaton CB, Buka SL, et al. Epigenomewide profiling of DNA methylation in paired samples of adipose tissue and blood. Epigenetics. 2016;11(3):227-36.

11. Rönn $T$, Volkov $P$, Gillberg $L$, Kokosar $M$, Perfilyev $A$, Jacobsen $A L$, et al. Impact of age, $\mathrm{BMI}$ and $\mathrm{HbA} 1 \mathrm{c}$ levels on the genome-wide DNA methylation and mRNA expression patterns in human adipose tissue and identification of epigenetic biomarkers in blood. Hum Mol Genet. 2015; 24(13):3792-813.

12. Trégouët D-A, Heath S, Saut N, Biron-Andreani C, Schved J-F, Pernod G, et al. Common susceptibility alleles are unlikely to contribute as strongly as the FV and ABO loci to VTE risk: results from a GWAS approach. Blood. 2009; 113(21):5298-303.

13. Wichmann H-E, Gieger C, Illig T, Group nftMKS. KORA-gen-resource for population genetics, controls and a broad spectrum of disease phenotypes. Das Gesundheitswesen. 2005;67(S 01:26-30.

14. Smith TG, Robbins PA, Ratcliffe PJ. The human side of hypoxia-inducible factor. Br J Haematol. 2008;141(3):325-34. 
15. Zhang H, Zhang G, Gonzalez FJ, Park SM, Cai D. Hypoxia-inducible factor directs POMC gene to mediate hypothalamic glucose sensing and energy balance regulation. PLoS Biology. 2011;9(7):e1001112.

16. Shin MK, Drager LF, Yao Q, Bevans-Fonti S, Yoo DY, Jun JC, et al. Metabolic consequences of high-fat diet are attenuated by suppression of HIF-1alpha. PloS one. 2012;7(10):e46562.

17. Pan H, Lin X, Wu Y, Chen L, Teh AL, Soh SE, et al. HIF3A association with adiposity: the story begins before birth. Epigenomics. 2015;7(6):937-50.

18. Huang T, Zheng Y, Qi Q, Xu M, Ley SH, Li Y, et al. DNA methylation variants at HIF3A locus, B-vitamin intake, and long-term weight change: gene-diet interactions in two U.S. cohorts. Diabetes. 2015;64(9):3146-54.

19. Richmond RC, Sharp GC, Ward ME, Fraser A, Lyttleton O, McArdle WL, et al. DNA methylation and BMl: investigating identified methylation sites at HIF3A in a causal framework. Diabetes. 2016;65(5):1231-44.

20. Lee S, Kim HJ, Han S, Jeon J-P, Park S-I, Yu H-Y, et al. Positive correlation of cg16672562 methylation with obesity-related traits in childhood obesity, and its independence with underlying HIF3A (hypoxia-inducible factor 3a) genetic background. Oncotarget. 2017;8(40):67473-81.

21. Wang S, Song J, Yang Y, Zhang Y, Wang H, Ma J. HIF3A DNA methylation is associated with childhood obesity and ALT. PloS one. 2016;10(12):e0145944.

22. Haertle L, El Hajj N, Dittrich M, Müller T, Nanda I, Lehnen H, et al. Epigenetic signatures of gestational diabetes mellitus on cord blood methylation. Clinical epigenetics. 2017;9(1):28.

23. Pasanen A, Heikkila M, Rautavuoma K, Hirsila M, Kivirikko Kl, Myllyharju J. Hypoxia-inducible factor (HIF)-3alpha is subject to extensive alternative splicing in human tissues and cancer cells and is regulated by HIF-1 but not HIF-2. Int J Biochem Cell Biol. 2010;42(7):1189-200.

24. Gutierrez-Arcelus M, Lappalainen T, Montgomery SB, Buil A, Ongen H, Yurovsky $A$, et al. Passive and active DNA methylation and the interplay with genetic variation in gene regulation. elife. 2013;2:e00523.

25. Al-Jameil N, Aziz Khan F, Fareed Khan M, Tabassum H. A brief overview of preeclampsia. J Clin Med Res. 2014;6(1):1-7.

26. Rolfo A, Many A, Racano A, Tal R, Tagliaferro A, letta F, et al. Abnormalities in oxygen sensing define early and late onset preeclampsia as distinct pathologies. PloS one. 2010;5(10):e13288.

27. Hatanaka M, Shimba S, Sakaue M, Kondo Y, Kagechika H, Kokame K, et al. Hypoxia-inducible factor-3a functions as an accelerator of 3 T3-L1 adipose differentiation. Biological and Pharmaceutical Bulletin. 2009;32(7):1166-72.

28. Heidbreder M, Qadri F, Jöhren O, Dendorfer A, Depping R, Fröhlich F, et al. Non-hypoxic induction of HIF-3a by 2-deoxy-d-glucose and insulin. Biochem Biophys Res Commun. 2007;352(2):437-43.

29. Mueller BR, Bale TL. Sex-specific programming of offspring emotionality after stress early in pregnancy. The Journal of Neuroscience. 2008;28(36):9055-65.

30. Clifton VL. Review: sex and the human placenta: mediating differential strategies of fetal growth and survival. Placenta. 2010;31(Supplement): S33-S9.

31. Rosenfeld CS. Sex-specific placental responses in fetal development. Endocrinology. 2015;156(10):3422-34

32. McRae AF, Powell JE, Henders AK, Bowdler L, Hemani G, Shah S, et al. Contribution of genetic variation to transgenerational inheritance of DNA methylation. Genome Biology. 2014;15(5):R73.

33. Gibbs JR, van der Brug MP, Hernandez DG, Traynor BJ, Nalls MA, Lai S-L, et al. Abundant quantitative trait loci exist for dna methylation and gene expression in human brain. PLoS Genetics. 2010;6(5):e1000952.

34. Gaunt TR, Shihab HA, Hemani G, Min JL, Woodward G, Lyttleton O, et al. Systematic identification of genetic influences on methylation across the human life course. Genome biology. 2016;17:61.

35. Lemire M, Zaidi SHE, Ban M, Ge B, Aïssi D, Germain M, et al. Long-range epigenetic regulation is conferred by genetic variation located at thousands of independent loci. Nature Communications. 2015;6:6326.

36. Drong AW, Nicholson G, Hedman ÅK, Meduri E, Grundberg E, Small KS, et al. The presence of methylation quantitative trait loci indicates a direct genetic influence on the level of DNA methylation in adipose tissue. PloS one. 2013; 8(2):e55923.

37. Gertz J, Varley KE, Reddy TE, Bowling KM, Pauli F, Parker SL, et al. Analysis of DNA methylation in a three-generation family reveals widespread genetic influence on epigenetic regulation. PLoS genetics. 2011;7(8):e1002228.

38. Bell JT, Pai AA, Pickrell JK, Gaffney DJ, Pique-Regi R, Degner JF, et al. DNA methylation patterns associate with genetic and gene expression variation in HapMap cell lines. Genome biology. 2011;12(1):R10.
39. Zhi D, Aslibekyan S, Irvin MR, Claas SA, Borecki IB, Ordovas JM, et al. SNPs located at CpG sites modulate genome-epigenome interaction. Epigenetics : official journal of the DNA Methylation. Society. 2013;8(8):802-6.

40. Consortium EP. An integrated encyclopedia of DNA elements in the human genome. Nature. 2012;489(7414):57.

41. Duan C. Hypoxia-inducible factor 3 biology: complexities and emerging themes. Am J Physiol Cell Physiol. 2016;310(4):C260-C9.

42. Vuillermin P, Saffery R, Allen KJ, Carlin JB, Tang ML, Ranganathan S, et al. Cohort profile: the Barwon Infant Study. Int J Epidemiol. 2015:44(4):1148-60.

43. Nankervis A, Mclntyre HD, Moses RG, Ross GP, Callaway LK. Testing for gestational diabetes mellitus in Australia. Diabetes care. 2013:36(5):e64

44. Tranquilli A, Dekker G, Magee L, Roberts J, Sibai B, Steyn W, et al. The classification, diagnosis and management of the hypertensive disorders of pregnancy: a revised statement from the ISSHP. Pregnancy hypertension. 2014:4(2):97.

45. Cole TJ, Williams AF, Wright CM. Revised birth centiles for weight, length and head circumference in the UK-WHO growth charts. Annals of human biology. 2011;38(1):7-11.

46. Group WMGRS. Reliability of anthropometric measurements in the WHO Multicentre Growth Reference Study. Acta paediatrica (Oslo, Norway: 1992) Supplement. 2006;450:38-46.

47. Mansell T, Novakovic B, Meyer B, Rzehak P, Vuillermin P, Ponsonby AL, et al. The effects of maternal anxiety during pregnancy on IGF2/H19 methylation in cord blood. Transl Psychiatry. 2016;6:e765.

48. Lam D, Ancelin ML, Ritchie K, Saffery R, Ryan J. DNA methylation and genetic variation of the angiotensin converting enzyme (ACE) in depression. Psychoneuroendocrinology. 2018;88:1-8.

49. Collier FM, Tang ML, Martino D, Saffery R, Carlin J, Jachno K, et al. The ontogeny of naïve and regulatory CD4+ T-cell subsets during the first postnatal year: a cohort study. Clin Transl Immunology. 2015;4(3):e34.

50. McCarthy S, Das S, Kretzschmar W, Delaneau O, Wood AR, Teumer A, et al. A reference panel of 64,976 haplotypes for genotype imputation. Nat Genet. 2016:48(10):1279-83.

51. Barrett JC, Fry B, Maller J, Daly MJ. Haploview: analysis and visualization of LD and haplotype maps. Bioinformatics. 2005;21(2):263-5.

\section{Publisher's Note}

Springer Nature remains neutral with regard to jurisdictional claims in published maps and institutional affiliations.

Ready to submit your research? Choose BMC and benefit from:

- fast, convenient online submission

- thorough peer review by experienced researchers in your field

- rapid publication on acceptance

- support for research data, including large and complex data types

- gold Open Access which fosters wider collaboration and increased citations

- maximum visibility for your research: over $100 \mathrm{M}$ website views per year

At $\mathrm{BMC}$, research is always in progress.

Learn more biomedcentral.com/submission 